\title{
Adherence to the Mediterranean Diet and Its Association with Body Composition and Physical Fitness in Spanish University Students
}

\author{
Ana Isabel Cobo-Cuenca ${ }^{1}\left(\right.$, Miriam Garrido-Miguel ${ }^{2, *}$, Alba Soriano-Cano ${ }^{2}$, \\ Asunción Ferri-Morales ${ }^{2}\left(\mathbb{D}\right.$, Vicente Martínez-Vizcaíno ${ }^{2,3}$ (D) and Noelia María Martín-Espinosa 4 \\ 1 Grupo Interdisciplinar en Cuidados IMCU, Universidad de Castilla la Mancha, 45071 Toledo, Spain; \\ Anaisabel.cobo@uclm.es \\ 2 Health and Social Research Center, Universidad de Castilla-La Mancha, 16071 Cuenca, Spain; \\ Alba.soriano@uclm.es (A.S.-C.); Asuncion.Ferri@uclm.es (A.F.-M.); vicente.martinez@uclm.es (V.M.-V.) \\ 3 Facultad de Ciencias de la Salud, Universidad Autónoma de Chile, 1101 Talca, Chile \\ 4 Facultad de Fisioterapia y Enfermería, Universidad de Castilla la Mancha, 45071 Toledo, Spain; \\ Noelia.martin@uclm.es \\ * Correspondence: miriam.garrido@uclm.es; Tel.: +34-969-170-091 (ext. 4686)
}

Received: 25 September 2019; Accepted: 15 November 2019; Published: 19 November 2019

\begin{abstract}
The aims of this study were to assess the association of adherence to the Mediterranean diet (MD) with physical fitness and body composition in Spanish university students and to determine the ability to predict the MD adherence of each Mediterranean Diet Adherence Screener (MEDAS) item. A cross-sectional study was performed involving 310 first-year university students. Adherence to the MD was evaluated with MEDAS-14 items. Anthropometric variables, body composition, and physical fitness were assessed. Muscle strength was determined based on handgrip strength and the standing long jump test. Cardiorespiratory fitness (CRF) was measured using the Course-Navette test. Only $24 \%$ of the university students had good adherence to the MD. The ANCOVA models showed a significant difference between participants with high adherence to the MD and those with medium and low adherence in CRF $(p=0.017)$ and dynamometry $(p=0.005)$. Logistic binary regression showed that consuming $>2$ vegetables/day (OR $=20.1 ; \mathrm{CI}: 10.1-30.1 ; p<0.001)$, using olive oil (OR $=10.6$; CI: 1.4-19.8; $p=0.021)$, consuming $<3$ commercial sweets/week $(\mathrm{OR}=10.1$; IC: $5.1-19.7 ; p<0.001)$, and consuming $\geq 3$ fruits/day (OR $=8.8 ; C I: 4.9-15.7 ; p<0.001$ ) were the items most associated with high adherence to the MD. In conclusion, a high level of adherence to the MD is associated with high-level muscular fitness and CRF in Spanish university students.
\end{abstract}

Keywords: Mediterranean diet; physical fitness; cardiovascular fitness; body composition

\section{Introduction}

The influence of diet on human health has aroused the interest of epidemiologists for hundreds of years. Among different dietary patterns, the Mediterranean diet (MD) has become very popular over the last decades, since the Seven Countries study reported the benefits of this diet for the prevention of a range of chronic disease outcomes [1]. Nowadays, some population-based studies and randomized trials have provided evidence suggesting that greater adherence to the MD reduces the risk of cardiovascular diseases, cancer, neurodegenerative diseases, diabetes, and all-cause mortality [2-5]. A meta-analysis demonstrated that a two-point increase in an adherence score was associated with a significant reduction in overall mortality and a reduced risk of cardiovascular diseases, cancer, and neurodegenerative diseases [6]. The MD represents a dietary pattern that incorporates healthy traditional eating habits of populations from countries surrounding the Mediterranean sea which 
includes high consumption of vegetables, fruit, legumes, nuts, beans, whole grains, grains, fish, and unsaturated fats, such as olive oil, and low consumption of red meat and dairy products, although the relationship between dairy consumption and health has been under discussion in recent years $[7,8]$.

In the last fifteen years, several validated scores have been used to measure the adherence to the $\mathrm{MD}$; although their reliability has been proven, more research is needed to determine their quality and cultural adaptation [9]. The Mediterranean Diet Adherence Screener (MEDAS-14 items) is a valid instrument for the estimation of adherence to the MD which has demonstrated itself to be sensitive to capturing the inverse association between $\mathrm{MD}$ and obesity indicators in high cardiovascular risk adults; moreover, this 14-item questionnaire requires less involvement from participants than the usual food frequency questionnaires and is less time demanding [10].

Physical fitness is robustly associated with a decrease in cardiovascular mortality as well as the risk of developing cardiovascular disease [11-13]. Recently, some studies have confirmed that higher levels of muscular strength are associated with a reduced risk of all-cause mortality in adults $[14,15]$, and studies have also shown the beneficial effect of higher muscular strength in both children and adolescents [16,17].

The transition to college or university is a critical period for young adults, who are often facing their first opportunity to make their own food decisions $[18,19]$. Sometimes, the lack of time and monetary concerns may determine the adoption of unhealthy eating habits and sedentary patterns [20,21], leading to increased weight $[22,23]$ which may persist during adulthood.

Although MD adherence has been studied in relation to physical fitness and body composition in different stages of life [24,25], research focused on the young adult group is scarce [26]. In this sense, the objectives of this study were (1) to estimate the prevalence of the adherence to the MD, (2) to assess the association of adherence to the MD with physical fitness and body composition in a sample of Spanish university students during their first year at university, and (3) to know the ability of each MEDAS item in the same sample to predict MD adherence.

\section{Materials and Methods}

\subsection{Study Design and Participants}

A cross-sectional observational study was performed with first-year university students, aged 18-30 years, during the 2017-2018 academic year belonging to the Faculties of Education, Nursing, Physiotherapy, and Social Work and Polytechnic, from the Albacete, Cuenca, and Toledo campuses of the University of Castilla-La Mancha, Spain. A total of 560 students were invited, and 310 (55.33\%) agreed to participate. Of these 310 participants, only 239 university students' total lean mass and total fat mass (by dual energy X-ray absorptiometry (DXA)) were measured. The participants included in this subsample did not differ in age, sex, or parental socioeconomic status from the whole sample of young adults participating in the study.

This report is part of the study: "Lifestyle, adiposity and vascular function in university students from Castilla-La Mancha, Spain". The Clinical Research Ethics Committee of the "Virgen de la Luz" of Cuenca approved the study, and the investigation adhered to the principles of the Declaration of Helsinki (REG: 2016jPI1116). Researchers explained the purpose of the study to eligible students: participating university students should not have any type of physical or mental disorder that prevented them from participating in physical measurements or completing questionnaires about lifestyle habits. Those who decided to participate in the study read and signed the informed consent form.

\subsection{Sample Size}

For a 95\% confidence level, the minimal sample size, calculated using Epidat 4.2, was 300 students considering an obesity prevalence of $23 \%$ [27], a precision of $5 \%$, and a $20 \%$ non-response rate. Taking as a sample frame the list of enrolments of these careers, a random 560 students were invited, from which 310 students agreed to participate. 


\subsection{Adherence to the Mediterranean Diet}

All participants completed two self-reported questionnaires: (a) The Mediterranean Diet Adherence Screener (MEDAS) [28], a Spanish-validated questionnaire that consisted of 14 items that included 12 questions about the frequency of food intake (olive oil, vegetables, fruit, red meat, animal fats, carbonated beverages, red wine, fish/seafood, nuts, commercial food, traditional dishes (with tomato sauce, garlic, onion, etc.) and two questions about the preferred cooking fat used and meat consumed. Each item was scored as zero or one, and the final score is the sum of each (0-14). (b) The Food-Frequency Questionnaire (FFQ) was used to determine the total intake of energy, carbohydrates, proteins, and fats [29]. The FFQ is a validated questionnaire with 137 items scored on a Likert scale with nine levels of frequency of consumption (never or almost never, 1-3 times per month, once per week, 2-4 times per week, 5-6 times per week, once per day, 2-3 times per day, 4-6 times per day, and more than six times per day).

\subsection{Anthropometrics and Body Composition Measures}

The students wore light clothes and were barefoot. Weight, height, and waist circumference were evaluated twice at a 5 min interval. Weight $(\mathrm{kg})$ was obtained using a SECA-7 scale. Height was measured using a wall-mounted stadiometer (Seca222, Vogel and Halke) with participants barefoot and standing against the wall with their chin parallel to the floor. Body mass index (BMI) (weight $(\mathrm{kg}) /$ height $\left.\left(\mathrm{m}^{2}\right)\right)$ was categorized into three groups: normal weight $(18.5 \leq \mathrm{BMI} \leq 24.9)$, overweight $(25 \leq \mathrm{BMI} \leq 29.9)$, and obesity (BMI $\geq 30)$ [30]. Waist circumference $(\mathrm{cm})$ was measured at the end of exhalation in the middle point between the costal margin and iliac crest. Body fat percentage (BF\%), total fat mass $(\mathrm{kg})$, and total lean mass $(\mathrm{kg})$ were obtained using dual-energy X-ray absorptiometry (DEXA) (Lunar iDXA, GE Medical Systems Lunar, Madison, WI 53718, USA). The students were scanned in a supine decubital position. All scans were performed at high resolution by two trained researchers.

\subsection{Sociodemographic and Lifestyle Variables}

For each enrolled student, a self-reported questionnaire including the following items was used: (1) Type of housing: family dwelling; university residence or shared apartment. (2) Lifestyle smoking habit, categorized as: non-smoker, who has not smoked more than 100 cigarettes in their lifetime and does not currently smoke; ex-smoker, who has smoked more than 100 cigarettes in their lifetime but has not smoked in the last 28 days; smoker, who smokes regularly. (3) Lifestyle alcohol habits: Never, 1-4 times a month, and $\geq 2$ times a week.

\subsection{Physical Fitness}

\subsubsection{Muscle Strength}

Handgrip strength was evaluated using a dynamometer (TKK 5401 Grip-D; Takey, Tokyo, Japan) and was adjusted for the sex and hand size of each participant. Lower limb strength was measured using the standing long jump test; students jumped from a starting line with both feet aligned. Both tests were performed twice, and the data from the best trial were recorded. With the data of the two strength tests, a strength index that consisted of the sum of the standardized z-scores of handgrip/weight and standing long jump was calculated. The best score recorded was used for analysis.

\subsubsection{Cardiorespiratory Fitness (CRF)}

Cardiorespiratory fitness (CRF) was measured using the Course Navette test (20 m shuttle run test (20 $\mathrm{m} \mathrm{SRT)).} \mathrm{Participants} \mathrm{had} \mathrm{to} \mathrm{run} \mathrm{between} \mathrm{two} \mathrm{lines} \mathrm{separated} \mathrm{by} \mathrm{a} \mathrm{distance} \mathrm{of} 20 \mathrm{~m}$, while keeping the rhythm of audio signals emitted by a pre-recorded compact disc. The initial speed was $8.5 \mathrm{~km} / \mathrm{h}$, which increased by $0.5 \mathrm{~km} / \mathrm{h}$ every minute. Participants were encouraged to continue running as long as possible during the course of the test. We recorded the last half stage completed as an indicator of 
their CRF. Leger's formula was used to obtain estimations of submaximal oxygen consumption (VO2) $(31.025+(3.238 \times$ velocity $)-(3.248 \times$ age $)+(0.1536 \times$ age $\times$ velocity $))[31]$.

\subsubsection{Physical Activity}

The total amount of physical activity was objectively measured using GENEActive accelerometers (ActivInsights). Participants wore these devices set at a fixed frequency of $30.0 \mathrm{~Hz}$ on their wrists for 7 consecutive days (including nights) for collecting raw acceleration data measured in " $\mathrm{g}$ " for each movement axis ( $\mathrm{x}, \mathrm{y}$, and $\mathrm{z})$, in order to estimate the participants' physical activity. We considered as valid measurements those of $\geq 5$ days, including 1 weekend day. For this study, mean total minutes/day of physical activity was estimated [32].

\subsection{Statistical Analysis}

For a descriptive analysis of the quantitative variables, the mean (m) and standard deviation (SD) were calculated; for categorical variables, count $(n)$ and percentages (\%) were used. We also compared proportions of categorical variables using chi-squared tests for contingency tables and student's $t$-test for quantitative variables.

The MEDAS scores were categorized as low adherence $(<9)$ and good adherence to the Mediterranean dietary pattern. Since recent studies have suggested that the relationship between adherence to the MD and cardiometabolic risk might have a linear relationship, we also categorized the adherence to the MD into three levels of adherence (low, first quartile; medium, second and third quartiles; and high, fourth quartile). To test mean differences in energy intake, body composition and fitness parameters by categories of MEDAS, ANCOVA models were calculated. Sex, age, smoking habits, alcohol habits, and type of housing were included as covariates in Model 1. Pairwise post-hoc comparisons were examined using the Bonferroni test

To quantify the predictive ability of each MEDAS item for adherence to the Mediterranean dietary pattern, logistic binary regression models were estimated using adherence to MD (low/good) as a dependent variable and each of the items of adherence to the MD questionnaire as independent variables. Statistical significance was set at $p<0.05$. We used the program IBM SPSS Statistics version 24 (IBM Corp., Armonk, NY, USA).

\section{Results}

The sample consisted of 310 students, $64.5 \%$ (202) of whom were women. The average age was 20.9 (SD 2.5) years. Weight, height, and total lean mass were significantly higher in men than in women $(p<0.001)$. The mean BF\%, total fat mass, and BMI were higher in women than in men $(p<0.001)$. A total of $72.8 \%$ of the participants were categorized as normal weight and $21.4 \%$ were categorized as overweight $(26.2 \%$ in men, $19.2 \%$ in women). Men showed higher muscular fitness z-scores and aerobic capacity $\left(\mathrm{VO}_{2}\right.$ max) than women (Table 1$)$.

Table 2 shows the agreement with the dietary recommendations based on 14 items from the FFQ and cooking preferences (questionnaire of Mediterranean diet adherence) by sex [28]. A very low adherence was observed in each of the items of the MEDAS questionnaire (14 items).

Table 3 (model 0 ) shows that participants classified as having high adherence to the Mediterranean dietary pattern showed higher weight, BMI, and total lean mass $(\mathrm{kg})$ than participants with medium and low adherence $(p<0.05)$. In addition, they also showed significantly higher CRF, z-scores of muscular strength index, handgrip strength $(\mathrm{kg})$, and standing long jump $(\mathrm{cm})$ than participants with medium and low adherence. Finally, the dietary pattern of those with higher adherence to the MD contained a higher percentage of protein and a lower percentage of fat than those with medium and low adherence $(p<0.05)$. However, the differences with BMI, total lean mass, and standing long jump did not reach statistical significance when controlling for potential confounders (model 1).

Table 4 shows, in a logistic binary regression model, that 12 of the 14 items of the MEDAS score were associated with a high adherence to the Mediterranean dietary pattern. To consume $>2$ servings 
of vegetables per day (OR = 20.1; CI: 10.1-30.1; $p<0.001)$, to use olive oil (OR = 10.6; CI: 1.4-19.8; $p=0.021)$, to consume $<3$ commercial sweets or pastries per week (OR $=10.1 ; \mathrm{IC}: 5.1-19.7 ; p<0.001)$, and to consume three or more fruits per day (OR $=8.8$; CI: 4.9-15.7; $p<0.001)$ were the items more closely associated with high MD adherence. Only the following two items were not significantly associated with the Mediterranean dietary pattern: "How many servings of butter, margarine, or cream do you consume per day?" and "How many sweet or carbonated beverages do you drink per day?".

Table 1. Descriptive characteristics of the study sample by sex.

\begin{tabular}{|c|c|c|c|c|}
\hline & All $(n=310)$ & $\operatorname{Men}(n=108)$ & Women $(n=202)$ & $p^{*}$ \\
\hline Age (years) & 20.9 (SD 2.5) & $21.1(\mathrm{SD} 2.8)$ & 20.7 (SD 2.3) & 0.146 \\
\hline Weight (Kg) & $65.4(\mathrm{SD} 12.3)$ & 72.6 (SD 10.99) & $61.4(\mathrm{SD} 11.1)$ & $<0.001$ \\
\hline Height $(\mathrm{cm})$ & $167.3(\mathrm{SD} 8.6)$ & $175.3(\mathrm{SD} 7.0)$ & $162.9(\mathrm{SD} 5.8)$ & $<0.001$ \\
\hline Waist circumference $(\mathrm{cm})$ & 78.9 (SD 9.3) & 83.0 (SD 7.9) & 76.6 (SD 9.2) & $<0.001$ \\
\hline$\%$ Fat mass ${ }^{a}$ & 29.3 (SD 9.0) & 20.5 (SD 6.3) & 33.7 (SD 6.7) & $<0.001$ \\
\hline Total fat mass $(\mathrm{Kg})^{\mathrm{a}}$ & 18.7 (SD 7.9) & 14.4 (SD 5.9) & 20.8 (SD 7.9) & $<0.001$ \\
\hline Total lean mass $(\mathrm{Kg})^{a}$ & 43.0 (SD 9.3) & $53.5(\mathrm{SD} 6.831)$ & 37.8 (SD 4.9) & $<0.001$ \\
\hline BMI $\left(\mathrm{Kg} / \mathrm{m}^{2}\right)$ & $23.3(\mathrm{SD} 3.5)$ & $23.5(\mathrm{SD} 3.03)$ & $23.1(\mathrm{SD} 3.8)$ & 0.269 \\
\hline Normal weight (\%) & 72.8 & 71.4 & 75.1 & \\
\hline Overweight $(\%)$ & 21.4 & 26.2 & 19.2 & 0.140 \\
\hline Obesity $(\%)$ & 4.4 & 2.4 & 5.7 & \\
\hline 20 m SRT (stages) & $5.8(\mathrm{SD} 2.6)$ & 7.0 (SD 1.9) & $3.8(\mathrm{SD} 1.4)$ & $<0.001$ \\
\hline $\mathrm{CRF}\left(\mathrm{VO}_{2}\right.$ max estimate $)$ & 33.4 (SD 9.9) & $40.4(\mathrm{SD} 8.9)$ & $28.7(\mathrm{SD} 7.6)$ & $<0.001$ \\
\hline Muscle strength $(\mathrm{cm} / \mathrm{Kg})^{\mathrm{b}}$ & 0.013 (SD 1.7) & 1.523 (SD 1.2) & $-1.050(\mathrm{SD} 1.2)$ & $<0.001$ \\
\hline Standing long jump (cm) & $161.2(\mathrm{SD} 43.7)$ & 195.4 (SD 31.9) & 136.8 (SD 33.5) & $<0.001$ \\
\hline Dynamometry $(\mathrm{kg})$ & 30.4 (SD 9.5) & $39.2(\mathrm{SD} 7.7)$ & $24.4(\mathrm{SD} 4.7)$ & $<0.001$ \\
\hline Physical activity (min/day) & $223.1(\mathrm{SD} 65.3)$ & $221.4($ SD 76.9) & $223.6(\mathrm{SD} 67.6)$ & 0.880 \\
\hline EI (Kcal) & 2795.7 (SD 1804.7) & 2865.9 (SD 1287.0) & 2757.6 (SD 2033.2) & 0.590 \\
\hline Carbohydrate (\% EI) & $43.0(\mathrm{SD} 7.1)$ & $43.1(\mathrm{SD} 6.6)$ & $42.9(\mathrm{SD} 7.3)$ & 0.852 \\
\hline Protein $(\%$ EI) & $17.4(\mathrm{SD} 3.4)$ & $17.4(\mathrm{SD} 3.2)$ & $17.5(\mathrm{SD} 3.6)$ & 0.749 \\
\hline Fat $(\% \mathrm{EI})$ & 38.2 (SD 6.2) & 37.9 (SD 5.9) & 38.3 (SD 6.3) & 0.578 \\
\hline \multicolumn{5}{|l|}{ Lifestyle smoking habits (\%) } \\
\hline Non-smoker & 81.3 & 82 & 80.9 & \multirow{3}{*}{0.580} \\
\hline Ex-smoker & 4.5 & 5.7 & 3.9 & \\
\hline Smoker & 14.2 & 12.3 & 15.2 & \\
\hline \multicolumn{5}{|l|}{ Lifestyle alcohol habits (\%) } \\
\hline Never & 10.1 & 12.7 & 8.6 & \multirow{3}{*}{0.173} \\
\hline 1-4 times a month & 88.2 & 84.1 & 90.6 & \\
\hline$\geq 2$ times a week & 1.7 & 3.2 & 0.9 & \\
\hline \multicolumn{5}{|l|}{ Type of housing (\%) } \\
\hline Family dwelling & 41.3 & 50.4 & 36.4 & \multirow{3}{*}{0.019} \\
\hline University residence & 19.8 & 19.7 & 19.9 & \\
\hline Shared apartment & 38.8 & 29.9 & 43.7 & \\
\hline \multicolumn{5}{|c|}{ Adherence Mediterranean diet (\%) } \\
\hline Low adherence & 65.4 & 70.4 & 79.0 & \multirow[t]{2}{*}{0.090} \\
\hline Good adherence & 24.0 & 29.6 & 21.0 & \\
\hline Total Mediterranean diet scores & $7.0(\mathrm{SD} 2.0)$ & $7.2(\mathrm{SD} 1.9)$ & $6.9(\mathrm{SD} 2.0)$ & 0.214 \\
\hline
\end{tabular}

Values are the mean and SD. Bold values indicate statistical significance $p \leq 0.05$. BMI $=$ body mass index, $\mathrm{EI}=$ energy intake. ${ }^{\text {a }}$ Subsample, $n=239 .{ }^{\mathrm{b}}$ Sum of the standardized z-score of dynamometry/weight and standing long jump. * Student's $t$-tests (continuous variables) or chi-squared tests (categorical variables). 
Table 2. Agreement with the recommendations based on each item of the Mediterranean Diet Adherence Screener (MEDAS-14 item score), by sex.

\begin{tabular}{|c|c|c|c|c|c|c|}
\hline & \multirow[t]{2}{*}{ Recommendation } & \multicolumn{2}{|c|}{$\begin{array}{l}\text { Agreement with the } \\
\text { Recommendation }\end{array}$} & \multicolumn{2}{|c|}{$\begin{array}{l}\text { No Agreement with } \\
\text { the Recommendation }\end{array}$} & \multirow[t]{2}{*}{$\begin{array}{l}p \text {-Value } \\
\left(\mathrm{X}^{2}\right)\end{array}$} \\
\hline & & Men & Women & Men & Women & \\
\hline $\begin{array}{l}\text { 1. Do you use olive oil as a main } \\
\text { culinary fat? }\end{array}$ & Yes & 92.1 & 90.1 & 7.9 & 9.9 & 0.530 \\
\hline $\begin{array}{l}\text { 2. How much olive oil do you } \\
\text { consume in a given day (including } \\
\text { oil used for frying, salads, } \\
\text { out-of-house meals, etc.)? }\end{array}$ & $\geq 4$ tbsp & 39.7 & 28.9 & 60.3 & 71.1 & 0.037 \\
\hline $\begin{array}{l}\text { 3. How many vegetable servings } \\
\text { do you consume per day? ( } 1 \\
\text { serving: } 200 \mathrm{~g} \text { (consider side dishes } \\
\text { as half a serving)) }\end{array}$ & $\begin{array}{l}\geq 2(\geq 1 \text { portion } \\
\text { raw or as a salad })\end{array}$ & 33.1 & 40.5 & 66.9 & 59.5 & 0.164 \\
\hline $\begin{array}{l}\text { 4. How many fruit units (including } \\
\text { natural fruit juices) do you } \\
\text { consume per day? }\end{array}$ & $\geq 3$ & 37.8 & 26.2 & 62.2 & 73.8 & 0.022 \\
\hline $\begin{array}{l}\text { 5. How many servings of red meat, } \\
\text { hamburger, or meat products (ham, } \\
\text { sausage, etc.) do you consume per } \\
\text { day? ( } 1 \text { serving: } 100-150 \mathrm{~g} \text { ) }\end{array}$ & $<1$ & 47.6 & 61.4 & 52.4 & 38.6 & 0.012 \\
\hline $\begin{array}{l}\text { 6. How many servings of butter, } \\
\text { margarine, or cream do you } \\
\text { consume per day? ( } 1 \text { serving: } 12 \text { g) }\end{array}$ & $<1$ & 94.4 & 95.7 & 5.6 & 4.3 & 0.585 \\
\hline $\begin{array}{l}\text { 7. How many sweet or carbonated } \\
\text { beverages do you drink per day? }\end{array}$ & $<1$ & 88.8 & 94.4 & 11.2 & 5.6 & 0.062 \\
\hline $\begin{array}{l}\text { 8. How much wine do you drink } \\
\text { per week? }\end{array}$ & $\geq 7$ glasses & 2.6 & 1.4 & 97.4 & 98.6 & 0.460 \\
\hline $\begin{array}{l}\text { 9. How many servings of legumes } \\
\text { do you consume per week? (1 } \\
\text { serving: } 150 \mathrm{~g})\end{array}$ & $\geq 3$ & 38.9 & 32.2 & 61.1 & 67.7 & 0.213 \\
\hline $\begin{array}{l}\text { 10. How many servings of fish or } \\
\text { shellfish do you consume per } \\
\text { week? ( } 1 \text { serving } 100-150 \mathrm{~g} \text { of fish } \\
\text { or } 4-5 \text { units or } 200 \mathrm{~g} \text { of shellfish) }\end{array}$ & $\geq 3$ & 29.6 & 32.3 & 70.4 & 67.7 & 0.596 \\
\hline $\begin{array}{l}\text { 11. How many times per week do } \\
\text { you consume commercial sweets or } \\
\text { pastries (not homemade), such as } \\
\text { cakes, cookies, biscuits, or custard? }\end{array}$ & $<3$ & 46.8 & 47.2 & 53.2 & 52.8 & 0.948 \\
\hline $\begin{array}{l}\text { 12. How many servings of nuts } \\
\text { (including peanuts) do you } \\
\text { consume per week? } \\
(1 \text { serving } 30 \mathrm{~g} \text { ) }\end{array}$ & $\geq 3$ & 26.2 & 18.0 & 73.8 & 82.0 & 0.069 \\
\hline $\begin{array}{l}\text { 13. Do you preferentially consume } \\
\text { chicken, turkey, or rabbit meat } \\
\text { instead of veal, pork, hamburger, } \\
\text { or sausage? }\end{array}$ & Yes & 70.1 & 70.4 & 29.9 & 29.6 & 0.951 \\
\hline $\begin{array}{l}\text { 14. How many times per week do } \\
\text { you consume vegetables, pasta, } \\
\text { rice, or other dishes seasoned with } \\
\text { sofrito (sauce made with tomato } \\
\text { and onion, leek, or garlic and } \\
\text { simmered with olive oil)? }\end{array}$ & $\geq 2$ & 68.5 & 62.1 & 31.5 & 37.9 & 0.224 \\
\hline
\end{tabular}


Table 3. The ANCOVA models comparing means of energy intake, body composition, and fitness parameters by MESA categories.

\begin{tabular}{|c|c|c|c|c|}
\hline & $\begin{array}{l}\text { Low Adherence } \\
(n=74)\end{array}$ & $\begin{array}{l}\text { Medium Adherence } \\
(n=160)\end{array}$ & $\begin{array}{l}\text { High Adherence } \\
(n=76)\end{array}$ & $p$ \\
\hline \multicolumn{5}{|l|}{ Model 0} \\
\hline Weight (Kg) & $62.9(\mathrm{SD} 12.1)$ & 64.3 (SD11.9) & $67.6(\mathrm{SD} 11.4)^{\mathrm{c}}$ & 0.047 \\
\hline Height (cm) & 166.3 (SD8.5) & 167.7 (SD9.1) & $168.6(\mathrm{SD} 8.4)$ & 0.282 \\
\hline $\mathrm{BMI}\left(\mathrm{Kg} / \mathrm{m}^{2}\right)$ & $22.6(\mathrm{SD} 3.1)$ & 22.7 (SD3.1) & 23.8 (SD3.8) & 0.050 \\
\hline Waist circumference $(\mathrm{cm})$ & 77.4 (SD9.4) & 78.0 (SD8.7) & 80.1 (SD9.1) & 0.419 \\
\hline $\begin{array}{l}\text { Waist circumference }(\mathrm{cm}) / \text { height } \\
(\mathrm{cm}) \text { index }\end{array}$ & 0.465 (SD0.04) & 0.465 (SD0.04) & 0.475 (SD0.05) & 0.297 \\
\hline$\%$ Fat mass & 28.9 (SD9.2) & 28.9 (SD9.6) & 28.9 (SD10.2) & 0.999 \\
\hline Total fat mass $(\mathrm{Kg})^{\mathrm{a}, *}$ & 17.9 (SD6.3) & 18.1 (SD6.1) & 18.2 (SD6.6) & 0.417 \\
\hline Total lean mass $(\mathrm{Kg})^{\mathrm{a}, *}$ & $42.2(\mathrm{SD} 6.0)$ & 42.3 (SD6.0) & $45.2\left(\mathrm{SD} 6.4^{\mathrm{c}}\right)$ & $<0.001$ \\
\hline 20 m SRT (stages) & $5.4(\mathrm{SD} 2.4)$ & $5.6(\mathrm{SD} 2.5)$ & $6.5\left(\mathrm{SD} 2.9^{\mathrm{c}}\right)$ & $<0.001$ \\
\hline $\mathrm{CRF}\left(\mathrm{VO}_{2}\right.$ max estimate) & 32.4 (SD9.0) & 32.8 (SD10.3) & $35.6\left(\mathrm{SD} 10.5^{\mathrm{c}}\right)$ & $<0.001$ \\
\hline Muscular strength index $b$ & $-0.223(\mathrm{SD} 1.6)$ & $-0.096(\mathrm{SD} 1.6)$ & $0.350\left(\mathrm{SD} 1.9^{\mathrm{c}}\right)$ & $<0.001$ \\
\hline Standing long jump $(\mathrm{cm}) *$ & $153.1(\mathrm{SD} 43.8)$ & $161.3(\mathrm{SD} 41.9)$ & $167.0\left(\mathrm{SD} 47.2^{\mathrm{c}}\right)$ & $<0.001$ \\
\hline Dynamometry $(\mathrm{kg}) *$ & 29.2 (SD9.5) & 29.3 (SD9.4) & $31.9\left(\mathrm{SD} 8.7^{\mathrm{c}}\right)$ & $<0.001$ \\
\hline Physical activity (min/day) & $216.6(\mathrm{SD} 58.8)$ & 220.18 (SD81.25) & $232.48(\mathrm{SD} 64.30)$ & 0.712 \\
\hline EI (Kcal) & 2570.7 (SD967.7) & 2883.9 (SD2301.0) & 2955.2 (SD1594.6) & 0.404 \\
\hline Carbohydrate (\% EI) & $42.9(\mathrm{SD} 6.7)$ & $42.6(\mathrm{SD} 6.9)$ & 43.8 (SD7.0) & 0.428 \\
\hline Protein $(\%$ EI) & $16.2(\mathrm{SD} 2.7)$ & $17.6(\mathrm{SD} 3.4)$ & $18.0\left(\mathrm{SD} 3.4^{\mathrm{c}}\right)$ & 0.002 \\
\hline Fat $(\% \mathrm{EI})$ & 39.5 (SD5.5) & 38.3 (SD6.1) & $36.7\left(\mathrm{SD} 6.3^{\mathrm{c}}\right)$ & 0.020 \\
\hline \multicolumn{5}{|l|}{ Model 1} \\
\hline Weight (Kg) & $65.0(\mathrm{SD} 11.3)$ & $66.3(\mathrm{SD} 12.0)$ & $68.0(\mathrm{SD} 11.4)$ & 0.258 \\
\hline Height (cm) & 168.8 (SD8.2) & 169.8 (SD9.0) & 169.6 (SD8.4) & 0.596 \\
\hline $\operatorname{BMI}\left(\mathrm{Kg} / \mathrm{m}^{2}\right)$ & $22.6(\mathrm{SD} 3.0)$ & 22.8 (SD3.2) & 23.4 (SD3.7) & 0.155 \\
\hline Waist circumference $(\mathrm{cm})$ & 78.7 (SD9.0) & 79.2 (SD8.8) & 80.2 (SD9.1) & 0.419 \\
\hline $\begin{array}{l}\text { Waist circumference }(\mathrm{cm}) / \text { height } \\
(\mathrm{cm}) \text { index }\end{array}$ & 0.466 (SD0.04) & 0.466 (SD0.04) & 0.474 (SD0.05) & 0.525 \\
\hline$\%$ Fat mass & 23.9 (SD9.9) & 23.9 (SD9.6) & 24.0 (SD10.1) & 0.997 \\
\hline Total fat mass $(\mathrm{Kg})^{a, *}$ & $16.0($ SD7.4) & 16.1 (SD7.2) & 17.1 (SD7.6) & 0.725 \\
\hline Total lean mass $(\mathrm{Kg})^{\mathrm{a}, *}$ & 42.5 (SD6.0) & 42.7 (SD6.0) & 43.1 (SD6.4) & 0.595 \\
\hline 20 m SRT (stages) & $5.7(\mathrm{SD} 2.4)$ & $6.1(\mathrm{SD} 2.6)$ & $6.6\left(\mathrm{SD} 2.6^{\mathrm{c}}\right)$ & 0.021 \\
\hline $\mathrm{CRF}\left(\mathrm{VO}_{2}\right.$ max estimate) & 33.4 (SD8.8) & 34.8 (SD10.3) & $36.5\left(\mathrm{SD} 10.5^{\mathrm{c}}\right)$ & 0.017 \\
\hline Muscular strength index $\mathrm{b}$ & 0.066 (SD1.6) & 0.173 (SD1.6) & $0.328(\mathrm{SD} 1.7)$ & 0.181 \\
\hline Standing long jump $(\mathrm{cm}) *$ & $158.6(\mathrm{SD} 43.8)$ & 165.3 (SD41.9) & 166.7 (SD47.2) & 0.366 \\
\hline Dynamometry $(\mathrm{kg}) *$ & 30.6 (SD9.0) & 30.1 (SD9.4) & $31.9\left(\mathrm{SD} 8.6^{\mathrm{d}}\right)$ & 0.005 \\
\hline Physical activity (min/day) & 198.1 (SD60.9) & $206.0($ SD84.2) & $242.5($ SD73.6) & 0.154 \\
\hline $\mathrm{EI}(\mathrm{Kcal})$ & 2576.7 (SD964.5) & 2889.7 (SD2340.0) & 3013.1 (SD1594.6) & 0.419 \\
\hline Carbohydrate (\% EI) & $42.9(\mathrm{SD} 6.8)$ & 42.7 (SD7.0) & 43.8 (SD7.0) & 0.566 \\
\hline Protein $(\%$ EI) & $16.0(\mathrm{SD} 2.7)$ & 17.5 (SD3.4) & $18.1\left(\mathrm{SD} 3.4^{\mathrm{c}}\right)$ & 0.002 \\
\hline Fat $(\% \mathrm{EI})$ & 39.5 (SD5.6) & 38.2 (SD6.2) & $36.7\left(\mathrm{SD} 6.3^{\mathrm{c}}\right)$ & 0.023 \\
\hline
\end{tabular}

Bold values indicate statistical significance $p \leq 0.05$. Abbreviations: $\mathrm{BMI}=$ body mass index; $\mathrm{EI}=$ energy intake. ${ }^{\text {a }}$ Subsample, $n=202$. ${ }^{\mathrm{b}}$ Sum of the standardized z-score of dynamometry/weight and standing long jump, ${ }^{\mathrm{c}}$ statistical significance $(p<0.05)$ in pairwise mean comparisons using Bonferroni post-hoc test: ${ }^{\mathrm{c}}$ low $<$ high; ${ }^{\mathrm{d}}$ medium $<$ high *adjusted for height. Model 0: crude data; Model 1: adjusted for sex, age, smoking habits, alcohol habits, and type of housing. 
Table 4. Logistic regression model predicting high adherence to the MD based on each item of the MD questionnaire.

\begin{tabular}{|c|c|c|c|}
\hline & Reference (1) & OR $(95 \% \mathrm{CI})$ & $p$-Value \\
\hline \multicolumn{4}{|l|}{ Questions from the MEDAS-14 } \\
\hline 1. Do you use olive oil as a main culinary fat? & Yes & $10.6(1.4-19.8)$ & 0.021 \\
\hline $\begin{array}{l}\text { 2. How much olive oil do you consume in a } \\
\text { given day (including oil used for frying, salads, } \\
\text { out-of-house meals, etc.)? }\end{array}$ & $\geq 4$ tbsp & $2.8(1.6-4.7)$ & $<0.001$ \\
\hline $\begin{array}{l}\text { 3. How many vegetable servings do you } \\
\text { consume per day? ( } 1 \text { serving: } 200 \mathrm{~g} \text { (consider } \\
\text { side dishes as half a serving)]) }\end{array}$ & $\begin{array}{l}\geq 2(\geq 1 \text { portion raw } \\
\quad \text { or as a salad })\end{array}$ & $20.1(10.1-30.1)$ & $<0.001$ \\
\hline $\begin{array}{l}\text { 4. How many fruit units (including natural fruit } \\
\text { juices) do you consume per day? }\end{array}$ & $\geq 3$ & $8.8(4.9-15.7)$ & $<0.001$ \\
\hline $\begin{array}{l}\text { 5. How many servings of red meat, hamburger, } \\
\text { or meat products (ham, sausage, etc.) do you } \\
\text { consume per day? ( } 1 \text { serving: } 100-150 \mathrm{~g})\end{array}$ & $<1$ & $2.8(1.5-4.9)$ & $<0.001$ \\
\hline $\begin{array}{l}\text { 6. How many servings of butter, margarine, or } \\
\text { cream do you consume per day? ( } 1 \text { serving: } 12 \mathrm{~g} \text { ) }\end{array}$ & $<1$ & $2.1(0.4-9.8)$ & 0.313 \\
\hline $\begin{array}{l}\text { 7. How many sweet or carbonated beverages do } \\
\text { you drink per day? }\end{array}$ & $<1$ & $6.6(0.8-50.3)$ & 0.068 \\
\hline 8. How much wine do you drink per week? & $\geq 7$ glasses & $6.4(1.1-35.9)$ & 0.034 \\
\hline $\begin{array}{l}\text { 9. How many servings of legumes do you } \\
\text { consume per week? ( } 1 \text { serving: } 150 \mathrm{~g} \text { ) }\end{array}$ & $\geq 3$ & $3.0(1.7-5.1)$ & $<0.001$ \\
\hline $\begin{array}{l}\text { 10. How many servings of fish or shellfish do } \\
\text { you consume per week? ( } 1 \text { serving } 100-150 \mathrm{~g} \text { of } \\
\text { fish or } 4-5 \text { units or } 200 \mathrm{~g} \text { of shellfish) }\end{array}$ & $\geq 3$ & $4.8(2.7-8.3)$ & $<0.001$ \\
\hline $\begin{array}{l}\text { 11. How many times per week do you consume } \\
\text { commercial sweets or pastries (not homemade), } \\
\text { such as cakes, cookies, biscuits, or custard? }\end{array}$ & $<3$ & $10.1(5.1-19.7)$ & $<0.001$ \\
\hline $\begin{array}{l}\text { 12. How many servings of nuts (including } \\
\text { peanuts) do you consume per week? (1 serving } \\
30 \mathrm{~g} \text { ) }\end{array}$ & $\geq 3$ & $5.3(2.9-9.4)$ & $<0.001$ \\
\hline $\begin{array}{l}\text { 13. Do you preferentially consume chicken, } \\
\text { turkey, or rabbit meat instead of veal, pork, } \\
\text { hamburger, or sausage? }\end{array}$ & Yes & $3.0(1.5-6.0)$ & 0.002 \\
\hline $\begin{array}{l}\text { 14. How many times per week do you consume } \\
\text { vegetables, pasta, rice, or other dishes seasoned } \\
\text { with sofrito (sauce made with tomato and onion, } \\
\text { leek, or garlic and simmered with olive oil)? }\end{array}$ & $\geq 2$ & $3.2(1.7-6.0)$ & $<0.001$ \\
\hline
\end{tabular}

Values are proportions. Bold values indicate statistical significance $p \leq 0.05$; Tbsp $=$ tablespoon.

\section{Discussion}

In this study, we evaluated the prevalence of the adherence to the MD and the relationship between adherence to the MD and physical fitness and body composition in a sample of Spanish university students during their first academic year. The main findings of this study were as follows: (1) the low prevalence of good adherence to the Mediterranean dietary pattern; (2) in those with good adherence to MD, the daily protein intake was higher than in those with medium-low adherence, while the fat intake was lower; (3) the good adherence group had significantly higher levels of physical fitness (CRF and muscular fitness); and (4) 12 of the 14 items of the MEDAS score were associated with a high adherence to the Mediterranean dietary pattern. 
A great variability in the dietary patterns of university students has been reported [33,34], even in studies conducted in the same country. In Mediterranean countries (Greece, Italy, Spain), adherence to the MD ranges from 20-30\% among students with lower prevalence rates [35-38] to more than $40 \%$ [39] or more than $70 \%$ [40] in those with higher prevalence rates. In our study, only $24 \%$ of the students scored as having good adherence to the MD. This variability could be because different questionnaires have been used to evaluate adherence to the MD. In a systematic review of the instruments for quantifying MD adherence, Zaragoza et al. [9] concluded that the psychometric properties and applicability parameters of most scores had not been tested; thus, only studies using the same tools could provide a good picture of the variability in the prevalence of MD adherence. Apart from this variability, in general, studies report low prevalence rates of adherence to the MD because young people tend to abandon the Mediterranean dietary pattern [41-44] and, in general, to move away from healthy lifestyle habits [45]. In a study on preferences when choosing food, students considered taste and pleasure as the main factors influencing their choice and that they did not take into account the importance of eating healthy food [46]. In addition, social pressure, the high cost of healthy food $[41,46]$, and short time for eating were decisive factors regarding the non-adherence to healthy diets [46].

In our sample, students with good adherence to the MD ingested more calories and protein and less fat than those with medium or low adherence. Similar results were found in other studies $[9,37,43]$. Furthermore, considering the food groups, the consumption of vegetables, olive oil, and fruits was positively associated with MD adherence, as has been described in other research $[9,43,47]$. The consumption of these foods is poor in the university population (possibly because of the price, lack of time, taste, etc.) both in Mediterranean and non-Mediterranean countries [33,34].

Although adherence to the MD diet was only acceptable, in our sample, more than $90 \%$ of participants used olive oil as a culinary fat, and less than $5 \%$ used another fat (margarine, butter, etc.). In Spain, the consumption of olive oil is very common, and olive oil is not expensive [40]. The use of oil olive as the main fat is attributable to the cultural heritage of Mediterranean countries. Olive oil reduces the incidence of cardiovascular diseases and increases HDL cholesterol, among other effects [48,49].

The influence of MD adherence on some anthropometric parameters is a controversial issue. Thus, while some studies suggest that adherence to this dietary pattern is associated with lower adiposity [50], others have reported that MD adherence did not predict weight status [51,52] or changes in WC [53]. In our sample, adherence to the MD was not associated with fat mass or WC, although those with higher adherence to the MD had higher levels of BMI and total lean. This fact may be explained by the fact that the MD is not a low caloric diet because of its high content of mono- and polyunsaturated fats and carbohydrates, all of which are potentially responsible for this weight increase [54]. In our study, at least part of this increase in lean mass may be attributable to practising more physical activity in the higher adherence to MD group. In addition, consistent evidence supports that muscle mass plays an important role in the prevention of some chronic diseases such as obesity and diabetes $[55,56]$.

Recent research conducted in adolescents and young adults supports the importance of muscular fitness in cardiovascular disease prevention. In a sample of 1248 college students, Ramirez-Velez et al. [26] found that those with optimal MD adherence and muscular fitness showed the healthiest cardiometabolic profile. Similar results were reported in another study including 2444 adolescents in which two components of physical fitness, aerobic capacity and muscular fitness, were examined [24]. In line with these findings and those of other studies [57-59], our data show that students with high MD adherence presented the highest levels of both cardiorespiratory and muscular fitness. If these results were confirmed in long-term follow-up studies, it would be very important, because the crucial role of physical fitness in reducing morbidity and mortality risk is not a debatable topic [16,58-65].

In our study, the analysis of the association of each individual item with the total MEDAS score shows that two items were not associated with the summative score: "How many servings of butter, margarine, or cream do you consume per day?" and "How many sweet or carbonated beverages do you drink per day?". In the Spanish context, the consumption of both food groups is not as common as 
in other countries; this may be the reason why these two items did not correlate with MD. According to the "Food Consumption in Spain 2018" report from the Spanish Ministry of Agriculture, Fisheries and Food [66], the aim of which is to determine the type of food consumed by the Spanish population, olive and sunflower oils are used for cooking in $64.9 \%$ and $32.1 \%$ of Spanish families, respectively, and margarine, butter, or cream represent only $1 \%$ of the total dairy intake. Likewise, the consumption of sweet or carbonated beverages in Spain is moderate and has displayed a declining trend during the last years, in such a way that in 2018 alone, Spanish people had reduced their consumption by $4 \%$ with respect to the previous year [66]. For this reason, although MEDAS is a validated questionnaire to determine the adherence to the MD, we believe it may be necessary to readjust this questionnaire to fit Spanish people's dietary patterns [9].

The strengths of this study include the novelty of combined associations of muscular fitness, CRF, body composition, and energy intake with MD in a large sample of college students and the ability to predict the MD adherence of each MEDAS item in the same sample.

There were some limitations of this study that should be acknowledged. First, its cross-sectional design prevents us from establishing cause-effect inferences. Second, the sample studied included only university students; thus, caution is necessary when making inferences about other age ranges. Third, of the 310 participants in our study, only in 239 university students' total lean mass and total fat mass (by dual energy X-ray absorptiometry (DXA)) were measured. Fourth, the use of self-reported dietary data could result in underreporting or unintentional measurement errors. In addition, the use of the FFQ-137 [29] and the MEDAS questionnaires [28] have only been validated in the elderly population; thus, the results of this study should be cautiously interpreted due to the potential bias that involves assuming that their validity and reliability could be extrapolated to a different age group in which these properties have never been proven.

\section{Conclusions}

Our results could be important from a public health point of view, since they reveal the low adherence of Spanish university students to the Mediterranean dietary patterns, and this could have long-term health consequences. Reinforcing the importance of Mediterranean dietary patterns for the health of young adults, our data support the association between MD adherence and both cardiorespiratory and muscular fitness. Finally, our data suggest that a MEDAS calibration that considers current Spanish dietary patterns could be suitable.

Author Contributions: Conceptualization, V.M.-V.; methodology, A.I.C.-C., M.G.-M., V.M.-V. and, N.M.M.-E.; software, M.G.-M.; validation, A.I.C.-C., M.G.-M., V.M.-V. and N.M.M.-E.; formal analysis, M.G.-M.; investigation, A.I.C.-C., M.G.-M., V.M.-V. and, N.M.M.-E.; resources, A.I.C.-C., M.G.-M., V.M.-V. and, N.M.M.-E.; data curation, A.I.C.-C., M.G.-M., V.M.-V. and, N.M.M.-E.; writing-original draft preparation, A.I.C.-C., M.G.-M., V.M.-V. and, N.M.M.-E.; writing-review and editing, A.I.C.-C., M.G.-M., V.M.-V. and, N.M.M.-E.; visualization, A.I.C.-C., M.G.-M., A.S.-C., A.F.-M., V.M.-V. and N.M.M.-E.; supervision, V.M.-V.; project administration, V.M.-V.

Funding: This research was funded by FEDER funds.

Acknowledgments: We thank all participants of the study.

Conflicts of Interest: The authors declare no conflict of interest.

\section{References}

1. Keys, A.; Mienotti, A.; Karvonen, M.J.; Aravanis, C.; Blackburn, H.; Buzina, R.; Djordjevic, B.S.; Dontas, A.S.; Fidanza, F.; Keys, M.H.; et al. The diet and 15-year death rate in the seven countries study. Am. J. Epidemiol. 1986, 124, 903-915. [CrossRef] [PubMed]

2. Willett, W.C. The Mediterranean diet: Science and practice. Public Health Nutr. 2006, 9, 105-110. [CrossRef] [PubMed]

3. Dinu, M.; Pagliai, G.; Casini, A.; Sofi, F. Mediterranean diet and multiple health outcomes: An umbrella review of meta-analyses of observational studies and randomised trials. Eur. J. Clin. Nutr. 2018, 72, 30. [CrossRef] [PubMed] 
4. Rees, K.; Takeda, A.; Martin, N.; Ellis, L.; Wijesekara, D.; Vepa, A.; Das, A.; Hartley, L.; Stranges, S. Mediterranean-style diet for the primary and secondary prevention of cardiovascular disease. Cochrane Database Syst. Rev. 2019, 3, CD009825. [CrossRef] [PubMed]

5. Schwingshackl, L.; Schwedhelm, C.; Galbete, C.; Hoffmann, G. Adherence to Mediterranean Diet and Risk of Cancer: An Updated Systematic Review and Meta-Analysis. Nutrients 2017, 9, E1063. [CrossRef] [PubMed]

6. Sofi, F.; Cesari, F.; Abbate, R.; Gensini, G.F.; Casini, A. Adherence to Mediterranean diet and health status: Meta-analysis. BMJ 2008, 337, a1344. [CrossRef]

7. Diez-Fernández, A.; Álvarez-Bueno, C.; Martínez-Vizcaíno, V.; Sotos-Prieto, M.; Recio-Rodríguez, J.; Cavero-Redondo, I. Total Dairy, Cheese and Milk Intake and Arterial Stiffness: A Systematic Review and Meta-Analysis of Cross-sectional Studies. Nutrients 2019, 11, 741. [CrossRef]

8. Cavero-Redondo, I.; Alvarez-Bueno, C.; Sotos-Prieto, M.; Gil, A.; Martinez-Vizcaino, V.; Ruiz, J.R. Milk and Dairy Product Consumption and Risk of Mortality: An Overview of Systematic Reviews and Meta-Analyses. Adv. Nutr. 2019, 10, S97-S104. [CrossRef]

9. Zaragoza-Martí, A.; Cabañero-Martínez, M.J.; Hurtado-Sánchez, J.A.; Laguna-Pérez, A.; Ferrer-Cascales, R. Evaluation of Mediterranean diet adherence scores: A systematic review. BMJ Open 2018, 8, e019033. [CrossRef]

10. Martínez-González, M.A.; García-Arellano, A.; Toledo, E.; Salas-Salvado, J.; Buil-Cosiales, P.; Corella, D.; Covas, M.I.; Schröder, H.; Arós, F.; Gómez-Gracia, E.; et al. A 14-item Mediterranean diet assessment tool and obesity indexes among high-risk subjects: The PREDIMED trial. PLoS ONE 2012, 7. [CrossRef]

11. Al-Mallah, M.H.; Sakr, S.; Al-Quinabet, A. Cardirespiratory fitness and cardiovascular disease prevention: An update. Curr. Atheroscler. Rep. 2018, 20, 1. [CrossRef] [PubMed]

12. Ozemek, C.; Laddu, D.R.; Lavie, C.; Claeys, H.; Kaminsky, L.A.; Ross, R.; Wisloff, U.; Arena, R.; Blair, S.N. An Update on the Role of Cardiorespiratory Fitness, Structured Exercise and Lifestyle Physical Activity in Preventing Cardiovascular Disease and Health Risk. Prog. Cardiovasc. Dis. 2018, 61, 484-490. [CrossRef] [PubMed]

13. Kaminsky, L.A.; Arena, R.; Ellingsen, Ø.; Harber, M.P.; Myers, J.; Ozemek, C.; Ross, R. Cardiorespiratory fitness and cardiovascular disease-The past, present, and future. Prog. Cardiovasc. Dis. 2019, 62, 86-93. [CrossRef] [PubMed]

14. Kodama, S.; Saito, K.; Tanaka, S.; Maki, M.; Yachi, Y.; Asumi, M.; Sugawara, A.; Totsuka, K.; Shimano, H.; Ohashi, Y.; et al. Cardiorespiratory fitness as a quantitative predictor of all-cause mortality and cardiovascular events in healthy men and women: A meta-analysis. JAMA 2009, 301, 2024-2035. [CrossRef] [PubMed]

15. García-Hermoso, A.; Cavero-Redondo, I.; Ramírez-Vélez, R.; Ruiz, J.R.; Ortega, F.B.; Lee, D.C.; Martínez-Vizcaíno, V. Muscular Strength as a Predictor of All-Cause Mortality in an Apparently Healthy Population: A Systematic Review and Meta-Analysis of Data From Approximately 2 Million Men and Women. Arch. Phys. Med. Rehabil. 2018, 99, 2100-2113.e5. [CrossRef]

16. Smith, J.J.; Eather, N.; Morgan, P.J.; Plotnikoff, R.C.; Faigenbaum, A.D.; Lubans, D.R. The health benefits of muscular fitness for children and adolescents: A systematic review and meta-analysis. Sports Med. 2014, 44, 1209-1223. [CrossRef]

17. Ortega, F.B.; Silventoinen, K.; Tynelius, P.; Rasmussen, F. Muscular strength in male adolescents and premature death: Cohort study of one million participants. BMJ 2012, 345, e7279. [CrossRef]

18. Deshpande, S.; Basil, M.D.; Basil, D.Z. Factors influencing healthy eating habits among college students: An application of the health belief model. Health Mark. Q. 2009, 26, 145-164. [CrossRef]

19. Papadaki, A.; Hondros, G.; Scott, J.; Kapsokefalou, M. Eating habits of university students living at, or away from home in Greece. Appetite 2007, 49, 169-176. [CrossRef]

20. Payne-Sturges, D.C.; Tjaden, A.; Caldeira, K.M.; Vincent, K.B.; Arria, A.M. Student Hunger on Campus: Food Insecurity Among College Students and Implications for Academic Institutions. Am. J. Health Promot. 2018, 32, 349-354. [CrossRef]

21. Martinez, S.M.; Grandner, M.A.; Nazmi, A.; Canedo, E.R.; Ritchie, L.D. Pathways from Food Insecurity to Health Outcomes among California University Students. Nutrients 2019, 11, E1419. [CrossRef] [PubMed]

22. Vella-Zarb, R.A.; Elgar, F.J. The 'freshman 5': A meta-analysis of weight gain in the freshman year of college. J. Am. Coll. Health 2009, 58, 161-166. [CrossRef] [PubMed]

23. Pope, L.; Hansen, D.; Harvey, J. Examining the Weight Trajectory of College Students. J. Nutr. Educ. Behav. 2017, 49, 137-141.e1. [CrossRef] 
24. Agostinis-Sobrinho, C.; Santos, R.; Rosário, R.; Moreira, C.; Lopes, L.; Mota, J.; Martinkenas, A.; García-Hermoso, A.; Correa-Bautista, J.E.; Ramírez-Vélez, R. Optimal Adherence to a Mediterranean Diet May Not Overcome the Deleterious Effects of Low Physical Fitness on Cardiovascular Disease Risk in Adolescents: A Cross-Sectional Pooled Analysis. Nutrients 2018, 10, E815. [CrossRef] [PubMed]

25. Labayen Goñi, I.; Arenaza, L.; Medrano, M.; García, N.; Cadenas-Sanchez, C.; Ortega, F.B. Associations between the adherence to the Mediterranean diet and cardiorespiratory fitness with total and central obesity in preschool children: The PREFIT project. Eur. J. Nutr. 2018, 57, 2975-2983. [CrossRef] [PubMed]

26. Ramírez-Vélez, R.; Correa-Bautista, J.E.; Ojeda-Pardo, M.L.; Sandoval-Cuellar, C.; García-Hermoso, A.; Carrillo, H.A.; González-Ruíz, K.; Prieto-Benavides, D.H.; Tordecilla-Sanders, A.; Martinkènas, A.; et al. Optimal Adherence to a Mediterranean Diet and High Muscular Fitness Are Associated with a Healthier Cardiometabolic Profile in Collegiate Students. Nutrients 2018, 10, E511. [CrossRef] [PubMed]

27. Gutiérrez-Fisac, J.L.; Guallar-Castillón, P.; León-Muñoz, L.M.; Graciani, A.; Banegas, J.R.; Rodríguez-Artalejo, F. Prevalence of general and abdominal obesity in the adult population of Spain, 2008-2010: The ENRICA study. Obes. Rev. 2012, 13, 388-392. [CrossRef]

28. Schröder, H.; Fitó, M.; Estruch, R.; Martínez-González, M.A.; Corella, D.; Salas-Salvadó, J.; Lamuela-Raventós, R.; Ros, E.; Salaverría, I.; Fiol, M.; et al. Screener Is Valid for Assessing Mediterranean Diet Adherence among Older Spanish Men and Women. J. Nutr. 2011, 41, 1140-1145. [CrossRef]

29. Fernández-Ballart, J.D.; Piñol, J.L.; Zazpe, I.; Corella, D.; Carrasco, P.; Toledo, E.; Pérez-Baurer, M.; Martínez-González, M.A.; Salas-Salvadó, J.; Martín-Moreno, J.M. Relative validity of a semi-quantitative food-frequency questionnaire in an elderly Mediterranean population of Spain. Br. J. Nutr. 2010, 103, 1808-1816. [CrossRef]

30. Organization WH. Obesity: Preventing and Managing the Global Epidemic; World Health Organization: Geneva, Switzerland, 2000.

31. Leger, L.A.; Mercier, D.; Gadoury, C.; Lambert, J. The multistage 20 meters shuttle run test for aerobic fitness. J. Sports Sci. 2007, 6, 93-101. [CrossRef]

32. Da Silva, I.C.; van Hees, V.T.; Ramires, V.V.; Knuth, A.G.; Bielemann, R.M.; Ekelund, U.; Brage, S.; Hallal, P.C. Physical activity levels in three Brazilian birth cohorts as assessed with raw triaxial wrist accelerometry. Int. J. Epidemiol. 2014, 43, 1959-1968. [CrossRef] [PubMed]

33. El Ansari, W.; Stock, C.; Mikolajczyk, R.T. Relationships between food consumption and living arrangements among university students in four European countries - a cross-sectional study. Nutr. J. 2012, 11, 28. [CrossRef] [PubMed]

34. Strawson, C.; Bell, R.; Downs, S.; Farmer, A.; Olstad, D.; Willows, N. Dietary patterns of female university students with nutrition education. Can. J. Diet. Pract. Res. 2013, 74, 138-142. [CrossRef] [PubMed]

35. Fiore, M.; Ledda, C.; Rapisarda, V.; Sentina, E.; Mauceri, C.; D'Agati, P.; Oliveri Conti, G.; Serra-Majem, L.; Ferrante, M. Medical school fails to improve Mediterranean diet adherence among medical students. Eur. J. Public Health 2015, 25, 1019-1023. [CrossRef] [PubMed]

36. Theodoridis, X.; Grammatikopoulou, M.G.; Gkiouras, K.; Papadopoulou, S.E.; Agorastou, T.; Gkika, I.; Maraki, M.I.; Dardavessis, T.; Chourdakis, M. Food insecurity and Mediterranean diet adherence among Greek university students. Nutr. Metab. Cardiovasc. Dis. 2018, 28, 477-485. [CrossRef] [PubMed]

37. García-Meseguer, M.J.; Burriel, F.C.; García, C.V.; Serrano-Urrea, R. Adherence to Mediterranean diet in a Spanish university population. Appetite 2014, 78, 156-164. [CrossRef] [PubMed]

38. Porto-Arias, J.J.; Lorenzo, T.; Lamas, A.; Regal, P.; Cardelle-Cobas, A.; Cepeda, A. Food patterns and nutritional assessment in Galician university students. J. Physiol. Biochem. 2018, 74, 119-126. [CrossRef]

39. Zurita-Ortega, F.; Román-Mata, S.; Chacón-Cuberos, R.; Castro-Sánchez, M.; Muros, J. Adherence to the Mediterranean Diet is associated with physical activity, self-concept and sociodemographic factors in university student. Nutrients 2018, 10, 966. [CrossRef]

40. Navarro-González, I.; López-Nicolás, R.; Rodríguez-Tadeo, A.; Ros-Berruezo, G.; Martínez-Marín, M.; Doménech-Asensi, G. Adherence to the Mediterranean diet by nursing students of Murcia (Spain). Nutr. Hosp. 2014, 30, 165-172. [CrossRef]

41. Bonaccio, M.; Bes-Rastrollo, M.; De Gaetano, G.; Lacoviello, L. Challenges to the Mediterranean diet at a time of economic crisis. Nutr. Metab. Cardiovasc. Dis. 2016, 26, 1057-1063. [CrossRef] 
42. Cabrera, S.G.; Fernández, N.H.; Hernández, C.R.; Nissensohn, M.; Román-Viñas, B.; Serra-Majem, L. KIDMED test; prevalence of low adherence to the Mediterranean Diet in children and young; a systematic review. Nutr. Hosp. 2016, 32, 2390-2399. [CrossRef]

43. Marventano, S.; Godos, J.; Platania, A.; Galvano, F.; Mistretta, A.; Grosso, G. Mediterranean diet adherence in the Mediterranean healthy eating, aging and lifestyle (MEAL) study cohort. Int. J. Food Sci. Nutr. 2018, 69, 100-107. [CrossRef] [PubMed]

44. Bibiloni, M.; González, M.; Julibert, A.; Llompart, I.; Pons, A.; Tur, J. Ten-year trends (1999-2010) of adherence to the Mediterranean diet among the Balearic Islands' adult population. Nutrients 2017, 9, 749. [CrossRef] [PubMed]

45. Stok, F.M.; Renner, B.; Clarys, P.; Deliens, T. Understanding Eating Behavior during the Transition from Adolescence to Young Adulthood: A Literature. Nutrients 2018, 10, 667. [CrossRef] [PubMed]

46. Sogari, G.; Velez-Argumedo, C.; Gómez, M.; Mora, C. College students and eating habits: A study using an ecological model for healthy behavior. Nutrients 2018, 10, 1823. [CrossRef] [PubMed]

47. Kyrkou, C.; Tsakoumaki, F.; Fotiou, M.; Dimitropoulou, A.; Symeonidou, M.; Menexes, G.; Michaelidou, A.M. Changing trends in nutritional behavior among university students in Greece, between 2006 and 2016. Nutrients 2018, 10, 64. [CrossRef]

48. Covas, M.I.; de la Torre, R.; Fitó, M. Virgin olive oil: A key food for cardiovascular risk protection. Br. J. Nutr. 2015, 113, S19-S28. [CrossRef]

49. Estruch, R.; Ros, E.; Salas-Salvadó, J.; Covas, M.I.; Corella, D.; Arós, F.; Gómez-Gracia, E.; Ruiz-Gutiérrez, V.; Fiol, M.; Lapetra, J.; et al. Primary prevention of cardiovascular disease with a Mediterranean diet. N. Engl. J. Med. 2013, 368, 1279-1290. [CrossRef]

50. Mistretta, A.; Marventano, S.; Antoci, M.; Cagnetti, A.; Giogianni, G.; Nolfo, F.; Rametta, S.; Pecora, G.; Marranzano, M. Mediterranean diet adherence and body composition among Southern Italian adolescents. Obes. Res. Clin. Pract. 2017, 11, 215-226. [CrossRef]

51. Sánchez-Villegas, A.; Bes-Rastrollo, M.; Martínez-González, M.A.; Serra-Majem, L. Adherence to a Mediterranean dietary pattern and weight gain in a follow-up study: The SUN cohort. Int. J. Obes. 2006, 30, 350-358. [CrossRef]

52. Archero, F.; Ricotti, R.; Solito, A.; Carrera, D.; Civello, F.; Di Bella, R.; Bellone, S.; Prodam, F. Adherence to the Mediterranean Diet among School Children and Adolescents Living in Northern Italy and Unhealthy Food Behaviors Associated to Overweight. Nutrients 2018, 10, E1322. [CrossRef] [PubMed]

53. Li, Y.; Roswall, N.; Ström, P.; Sandin, S.; Adami, H.O.; Weiderpass, E. Mediterranean and Nordic diet scores and long-term changes in body weight and waist circumference: Results from a large cohort study. Br. J. Nutr. 2015, 114, 2093-2102. [CrossRef] [PubMed]

54. Zappalà, G.; Buscemi, S.; Mulè, S.; La Verde, M.; D’Urso, M.; Corleo, D.; Marranzano, M. High adherence to Mediterranean diet, but not individual foods or nutrients, is associated with lower likelihood of being obese in a Mediterranean cohort. Eat. Weight Disord. 2018, 23, 605-614. [CrossRef]

55. Wolfe, R.R. The underappreciated role of muscle in health and disease. Am. J. Clin. Nutr. 2006, 84, 475-482. [CrossRef]

56. Stump, C.S.; Henriksen, E.J.; Wei, Y.; Sowers, J.R. The metabolic syndrome: Role of skeletal muscle metabolism. Ann. Med. 2006, 38, 389-402. [CrossRef]

57. Galan-Lopez, P.; Ries, F.; Gisladottir, T.; Domínguez, R.; Sánchez-Oliver, A.J. Healthy Lifestyle: Relationship between Mediterranean Diet, Body Composition and Physical Fitness in 13 to 16-Years Old Icelandic Students. Int. J. Environ. Res. Public Health. 2018, 15, E2632. [CrossRef]

58. Evaristo, O.S.; Moreira, C.; Lopes, L.; Abreu, S.; Agostinis-Sobrinho, C.; Oliveira-Santos, J.; Póvoas, S.; Oliveira, A.; Santos, R.; Mota, J. Associations between physical fitness and adherence to the Mediterranean diet with health-related quality of life in adolescents: Results from the LabMed Physical Activity Study. Eur. J. Public Health. 2018, 28, 631-635. [CrossRef]

59. Muros, J.J.; Cofre-Bolados, C.; Arriscado, D.; Zurita, F.; Knox, E. Mediterranean diet adherence is associated with lifestyle, physical fitness, and mental wellness among 10-y-olds in Chile. Nutrition 2017, 35, 87-92. [CrossRef]

60. Jochem, C.; Leitzmann, M.; Volaklis, K.; Aune, D.; Strasser, B. Association Between Muscular Strength and Mortality in Clinical Populations: A Systematic Review and Meta-Analysis. J. Am. Med. Dir. Assoc. 2019, 20, 1213-1223. [CrossRef] 
61. Wu, Y.; Wang, W.; Liu, T.; Zhang, D. Association of grip strength with risk of all-cause mortality, cardiovascular diseases, and cancer in community-dwelling populations: A meta-analysis of prospective cohort studies. J. Am. Med. Dir. Assoc. 2017, 18, 551.e17-551.e35. [CrossRef]

62. García-Hermoso, A.; Ramírez-Campillo, R.; Izquierdo, M. Is Muscular Fitness Associated with Future Health Benefits in Children and Adolescents? A Systematic Review and Meta-Analysis of Longitudinal Studies. Sports Med. 2019, 49, 1079-1094. [CrossRef] [PubMed]

63. Ross, R.; Blair, S.N.; Arena, R.; Church, T.S.; Després, J.P.; Franklin, B.A.; Haskell, W.L.; Kaminsky, L.A.; Levine, B.D.; Lavie, C.J.; et al. Importance of Assessing Cardiorespiratory Fitness in Clinical Practice: A Case for Fitness as a Clinical Vital Sign: A Scientific Statement From the American Heart Association. Circulation 2016, 134, e653-e699. [CrossRef] [PubMed]

64. Imboden, M.T.; Harber, M.P.; Whaley, M.H.; Finch, W.H.; Bishop, D.L.; Kaminsky, L.A. Cardiorespiratory Fitness and Mortality in Healthy Men and Women. J. Am. Coll. Cardiol. 2018, 72, 2283-2292. [CrossRef] [PubMed]

65. Laukkanen, J.A.; Zaccardi, F.; Khan, H.; Kurl, S.; Jae, S.Y.; Rauramaa, R. Long-term Change in Cardiorespiratory Fitness and All-Cause Mortality: A Population-Based Follow-up Study. Mayo Clin. Proc. 2016, 91, 1183-1188. [CrossRef]

66. Ministerio de Agricultura, Pesca y Alimentación. Informe del Consumo Alimentario en España 2018; Ministerio de Agricultura, Pesca y Alimentación: Madrid, Spain, 2019. Available online: https://www.mapa.gob.es/ images/es/20190807_informedeconsumo2018pdf_tcm30-512256.pdf (accessed on 29 August 2019).

(C) 2019 by the authors. Licensee MDPI, Basel, Switzerland. This article is an open access article distributed under the terms and conditions of the Creative Commons Attribution (CC BY) license (http://creativecommons.org/licenses/by/4.0/). 